CHAPTER V

THE THEORY OF GALAXY FORMATION AND LARGE-SCALE STRUCTURE 


\title{
GALAXY FORMATION: CONFRONTATION WITH OBSERVATIONS
}

\author{
Joseph Silk \\ Astronomy Department \\ University of California \\ Berkeley, CA 94720 \\ USA
}

\begin{abstract}
The implications for galaxy formation of inflationary cosmology are reviewed. In particular, I explore some implications of the hypothesis that galaxies form from adiabatic, gaussian density fluctuations in a cold dark matterdominated universe. Topics discussed include protogalaxies and the epoch of galaxy formation, Lyman alpha clouds, dark halos and dwarf galaxies. Finally I describe how environmental biasing may arise as a consequence of tidally induced star formation in protoclusters.
\end{abstract}

\section{INTRODUCTION}

A notable development in cosmology, and in particular, in galaxy formation theory, over the past few years has been the gradual emergence of a standard model. This is due in large part to the contribution from particle physics, which has provided a theoretical framework for describing the evolution of the very early universe. Without this input, the initial value problem, namely the unknown initial conditions that characterized the density fluctuations at early epochs, presented an insuperable obstacle. I am going to tell you a modern myth. It has one advantage over the myths of ancient times - it is falsifiable. This brings it into the realm of science. The myth begins a long, long time ago with the creation of the universe. Once upon a time there was nothing, nothing at all. Not space, nor even time. This was the realm of quantum gravity. Our understanding of quantum gravity is inadequate, at present, to describe the initial singularity predicted by the classical regime of the Big Bang theory, although recent developments in the theory of higher dimensional space-time descriptions of physics, such as superstrings, have persuaded many physicists around the world that development of a unified theory of quantum gravity is imminent. From the Planck time, $t_{p l} \sim 10^{-43} \mathrm{~s}$, onward, however, classical gravity suffices, and the very early universe provides 
a natural laboratory for studying the grand unification of the electro-weak and strong nuclear interactions.

As the universe expands and adiabatically cools, from an energy or temperature of about $10^{19} \mathrm{GeV}$ at the Planck time to about $10^{15} \mathrm{GeV}$, the symmetry is spontaneously broken, and the more familiar interaction strengths of the present universe emerge. The symmetry breaking is characterized by a first-order phase transition which triggers a brief period of exponential expansion or inflation (see e.g. Turner 1985 for a review of inflation). Preexisting inhomogeneities and anisotropies, of more or less generic type, are erased, while quantum fluctuations are amplified up to the horizon scale. Because of the nature of the free quantum fields, the fluctuations are gaussian: this is effectively guaranteed by the central limit theorem. Since the horizon inflates to a scale far larger than our present horizon, this means that we are left with a density fluctuation spectrum that can eventually power growth of large-scale structure on all observable scales. No preferred scale is selected for these fluctuations: one characterizes them by saying that the associated curvature fluctuations are of constant amplitude on all scales. Curvature is measured by the density fluctuation amplitude at horizon crossing, and one has the scale-invariant Zel'dovich-Harrison fluctuation spectrum $(\delta \rho / \rho)_{H} \approx$ constant. At the same time, the erasure of any preexisting global curvature by inflation means that the universe must be flat to a high degree of precision: specifically

$$
\Omega=1 \pm O(|\delta \rho / \rho|) .
$$

The density parameter $\Omega$ is defined to be the density expressed relative to the critical value for closure, $3 H_{o}^{2} / 8 \pi G$. Inflation thereby accounts for a paradox implicit in the Friedmann equation, which can be expressed in the form

$$
\Omega \approx 1+K t
$$

where $K$ is a constant of order unity that depends on the curvature of the universe. If today, at $t \approx 6 \times 10^{17} \mathrm{~s}, \Omega$ were equal, for example to 0.1 , one would then have great difficulty is understanding why, at $t_{p l}, \Omega$ must have been equal to unity to within about 60 decimal places. Inflation naturally accounts for this coincidence. While inflation was by no means inevitable, nor did it necessarily lead to an acceptable amplitude for the density fluctuations, it has resolved enough problems in our understanding of the very early universe for us to regard it as a yardstick by which any rival theory has to be compared. I shall therefore adopt it as the standard model in the ensuing discussion.

This standard model also incorporates two other successes that result from the application of particle physics to cosmology. These are baryon genesis and light element nucleosynthesis. The universe today consists predominantly of baryons, not antibaryons, and the baryon number, a conserved quantity, can be expressed 
as $n_{B} / n_{\gamma}=10^{-8} \Omega h^{2}$, where $h \equiv H_{o} / 100 \mathrm{~km}^{-1} \mathrm{~s}^{-1} \mathrm{Mpc}^{-1}$. This manifests itself at early epochs as a slight baryon assymmetry, and can be understood as arising from weakly $\mathrm{CP}$ and baryon number violating processes that occurred following the symmetry breaking phase when inflation ended and the universe reheated to very high temperature, in the range $10^{10} \mathrm{GeV}-10^{16} \mathrm{GeV}$. Since a universal baryon number results, this also means that one is unlikely to have had any entropy fluctuations: apart from one possible exception (in an axion-dominated universe), the surviving density fluctuations are adiabatic.

Primordial nucleosynthesis occurred much later, when weak interactions froze out at $T \sim 1 \mathrm{MeV}$, leaving a residual neutron abundance of about 10 percent. Most of these neutrons were subsequently synthesized into ${ }^{4} \mathrm{He}$ at $\mathrm{T} \sim 0.1 \mathrm{MeV}$, and traces of other light elements, most notably ${ }^{2} \mathrm{H},{ }^{3} \mathrm{He}$, and ${ }^{7} \mathrm{Li}$, were also produced. Remarkedly, these abundances agree with the observed abundances, to within uncertainties of corrections for galactic evolution contributions to light element synthesis (Yang et al. 1984). One critical parameter has to be adjusted, namely the baryon density, and

$$
\Omega_{b} h^{2} \approx 0.03
$$

is required for consistency. There are no other plausible sources for ${ }^{4} \mathrm{He}$ and ${ }^{2} \mathrm{H}$, both of which must be of pregalactic origin, and explaining their abundances to within one or two percent (in the case of ${ }^{4} \mathrm{He}$ ) or to within a factor of 2 (in the case of ${ }^{2} H$ ) by adjustment of one plausible parameter is a powerful argument for a big bang origin.

One may now summarize the standard model of the early universe: $\Omega=$ $1, \Omega_{b} \approx 0.1$, and adiabatic, gaussian density fluctuations with a scale-invariant spectrum. I add one more ingredient, namely cold dark matter. Dark matter is needed in order for $\Omega>>\Omega_{b}$, and cold dark matter, by which is meant any species of weakly interacting massive particle, provides a generic description. Such particles are plausible stable relics from supersymmetry: candidates include photinos, higgsinos, or scalar neutrinos. These heavy particles generally have mass of order $1 \mathrm{GeV}$, and so have negligible velocity dispersion when the universe first becomes matter dominated at $T \sim 10 \mathrm{eV}$ and effective fluctuation growth commences. Axions, while light, are non-relativistic when first produced at $T \gtrsim 10^{10} \mathrm{GeV}$, and so are also a form of cold dark matter.

I regard the principal alternative, hot dark matter, characterized either by a universe dominated by a light, $\sim 30 \mathrm{eV}$, neutrino or by adiabatic fluctuations in a baryon-dominated universe, as being less likely, both because of the recent experimental limits $\left(m_{\nu_{e}}<18 \mathrm{eV}\right)$ (Fritschi et al. 1986) on neutrino mass and because of theoretical objections to a hot dark matter-dominated universe that 
arise in simulations of the large-scale galaxy distribution (Frenk et al. 1983). Free streaming of hot dark matter suppresses fluctuation growth on mass scales below

$$
1.8 m_{p l}^{3} / m_{\nu}^{2} \sim 10^{15}\left(30 \mathrm{eV} / m_{\nu}\right)^{2} M_{\odot}
$$

and requires a top-down scenario for forming structure in the universe today in which cluster-mass structures anisotropically collapsed, pancaked, and fragmented into galaxies. The large-scale structure constraints from galaxy correlations mean that such theories fail to account for galaxy formation at the epoch when quasars are seen (to a redshift that now exceeds 4), unless some additional ingredient is added that allows galaxies to form prior to pancaking.

Cold dark matter provides a hierarchical or bottom-up scenario for the development of structure. Fluctuations grow first on small scales, and eventually develop on very large scales. The only free parameter is the initial amplitude of the Zel'dovich Harrison scale-invariant spectrum; the value required in order to fabricate large-scale structure by today is

$$
(\delta \rho / \rho)_{H} \sim 10^{-4} .
$$

Any smaller value would not generate sufficient structure; any larger value would generate excessive structure. For the moment, there is no natural explanation for the origin of the amplitude (5), although anthropic arguments have been given in the context of inflationary scenarios. The constraint (5) can be reinterpreted as a constraint on the potential that drives inflation, and presumably an explanation will eventually emerge from particle physics (Steinhardt and Turner 1984).

A unique fluctuation spectrum therefore arises in the standard model, and its amplitude is adjusted to yield the observed correlation length in the galaxy distribution. This in itself is a non-trivial adjustment, since there is a natural scale that is imprinted on the residual, post-recombination, fluctuation spectrum, namely the comoving horizon scale at equality of matter and radiation densities,

$$
L_{e q} \approx 10\left(\Omega h^{2}\right)^{-1} \mathrm{Mpc} .
$$

Small scales enter the horizon during radiation domination and undergo only logarithmic growth (Mészáros 1974); consequently the residual fluctuation spectrum is nearly flat at $L<<L_{e q}$, as expected for sub-horizon growth of $\delta \rho / \rho$ proportional to $t^{2 / 3}$ during the matter-dominated era.

Numerical simulations have provided a quantitative test of this theory. The slope of the simulated correlation function steepens with time: it only agrees with the observed function

$$
\xi(\nu)=\left(r_{o} / r\right)^{-1.8}, r_{o}=5 h^{-1} \mathrm{Mpc}
$$


at a unique epoch (or equivalently by today, for a specified Hubble constant). The standard model is found to give a good fit to the slope and amplitude of the observed galaxy correlations if galaxy formation occurred recently and $H_{o} \approx$ $50 \mathrm{~km} \mathrm{~s}^{-1} \mathrm{Mpc}^{-1}$, provided that an additional parameter is added in order to allow mass to be more uniformly distributed than the luminous galaxies (Davis et al. 1985).

This additional parameter is specified as follows. In addition to galaxy correlations, one also has dynamical information on galaxy peculiar motions. Regarding galaxies as test particles, these allow a determination of $\Omega_{d y n}$, which is measured on scales of up to $\sim 10 \mathrm{Mpc}$ to be

$$
\Omega_{d y_{n}} \approx 0.2( \pm 0.1) \text {. }
$$

To reconcile this with our standard model $(\Omega=1)$, the dominant mass contribution must be smoothly distributed on scales up to $\sim 10 h^{-1} \mathrm{Mpc}$. One can quantity this: the correlation length for the mass distribution must be between one-half and one-third of that observed for the galaxies. Then the measured $\Omega_{d y n}$, which for a given velocity measurement is inversely proportional to $\int_{0}^{r} \xi(r) r d r$, or the potential energy, can be reduced relative to $\Omega=1$ by about a factor of 3-10.

This renormalization of the underlying mass distribution relative to that of the galaxies is parametrized by the introduction of a biasing threshold (Bardeen et al. 1986). For initially gaussian fluctuations, the rare peaks are more clustered than the common peaks. Denoting a peak by $\nu \sigma$, where $\sigma$ is the rms density fluctuation amplitude, then imposition of a threshold for galaxy formation of $\nu \sigma$, such that galaxies only form in peaks greater than or equal to $\nu \sigma$, biases the correlation function: it is enhanced (Kaiser 1984; Politzer and Wise 1984) by a factor $\sim\left\{\exp \left[\nu^{2} \xi(r)\right]-1\right\} / \xi \approx \nu^{2}$. Galaxy formation at $2.5 \sigma$ peaks, with galaxies defined to be fluctuations filtered over a comoving scale of $0.5 h^{-1} \mathrm{Mpc}$, is consistent with the observed correlations and the number density of galaxies for the standard model. Filtering is necessary in order to smooth over the smaller peaks which, while containing less mass, would dominate number counts. One of the topics to be discussed below will be the physical origin of this biasing towards rare peaks.

There are additional hurdles in the realm of large-scale structure that the standard model of biased cold dark matter satisfactorily overcomes. The predicted cosmic microwave background fluctuations are low, with $\delta T / T \sim(3-5) \times 10^{-6}$, and below current upper limits of about $3 \times 10^{-5}$ (on a wide range of angular scales, from $1^{\prime}$ up to $90^{\circ}$ ) (Kaiser and Silk 1986). Large voids are produced in the simulated galaxy distribution as a consequence of the biasing threshold, and filaments and sheets of galaxies are present that resemble those observed (White 
et al. 1987). The frequency of rich clusters is comparable to that of the Abell clusters, when summed over richness class.

These are the principal successes arising from confrontation with observation on large scales. There are also difficulties, or potential difficulties, lurking ahead. The most serious of these concerns the cluster-cluster correlations. Clusters are themselves clustered, and the strength of this clustering is such that the correlation length is about five times larger than the galaxy correlation length (Bahcall and Soneira 1983). The standard model simulations produce some enhancements of cluster correlations, but the cluster correlation length is only enhanced by a factor of two relative to the galaxy correlation length (Barnes et al. 1985). However systematic errors in the cluster-cluster correlation function are very large, especially on large scales (Ling et al. 1986), and it is probably premature to reject the standard model on this account. A potentially even more serious problem for biased cold dark matter is the recent report of cosmic drift velocities of galaxy clusters over scales of up to $\sim 60 h^{-1} \mathrm{Mpc}$ that amount to $\sim 600 \mathrm{~km} \mathrm{~s}^{-1}$ (Dressler et al. 1987). The model predicts velocities of $\sim 100 \mathrm{~km} \mathrm{~s}^{-1}$ on such large scales: however, the reality of the cosmic drift velocities have yet to be definitively confirmed.

My philosophy is the following: the standard model of biased cold dark matter is aesthetically appealing, it has fewer free parameters than any other model of large-scale structure, and its predictions have been quantified in more detail than for any other models. The theory is a falsifiable theory. At present, the possible problems are minor compared to the advantages. I will take the attitude that the cluster-cluster correlations, for example, are a possibly major hurdle looming ahead for the standard model, but do not yet provide sufficient motivation to abandon cold dark matter. Of course, there is the likelihood that an improved model of large-scale structure would represent a variant on the standard model rather than being something radically different. In the absence of any alternative, we are surely justified in believing that the germ of the ultimate truth is already present in a model that is so well-motivated and has already met many challenges.

This review therefore considers galaxy formation in the context of the standard model, although many of the conclusions can readily be generalized to other models. I will emphasize observable implications of the standard model, including the epoch of protogalaxy formation and the role of Lyman alpha clouds and dark halos. In a final section, I will describe a biasing scheme and how environment plays an important role in determining galaxy morphology.

\section{PROTOGALAXIES}

Normalization of the large-scale structure to yield the observed galaxy correlation function fixes the rms mass density fluctuations today on a luminous galaxy 
filtering scale of $0.5 h^{-1} \mathrm{Mpc}$ such that collapse has occurred for $\nu \sigma$ density fluctuations, represented by a spherical "top-hat" model with density contrast 1.69 at collapse, at a redshift $z_{g}=2.5 \nu / 1.69-1=2.7$ for $\nu=2.5$. The number density of $2.5 \sigma$ fluctuations then agrees with the observed frequency of luminous galaxies. This normalization yields rms mass fluctuations $(\delta M / M)_{\mathrm{rms}} \approx 1$ at $3 \mathrm{~h}^{-1} \mathrm{Mpc}$ and corresponds to a correlation scale for the $2.5 \sigma$ fluctuations today of about $8 \mathrm{~h}^{-1}$ where the correlations have unit amplitude (Bardeen et al. 1986).

The obvious difficulty with this scheme is that primeval galaxies are few and far between. Searches in random fields have not found any out to a redshift of about 5, with Lyman alpha emission as the principal search criterion (Koo 1986). To compare properly theory with this observational constraint, one has to examine carefully the epoch of formation of the luminous cores of galaxies, rather than of their halos. In fact, in a hierarchical scheme such as that of cold dark matter, the cores of galaxies can form long before the associated halos (Silk and Wyse 1986).

To evaluate this quantitatively, one may evaluate the probability that within a specified filter scale $R_{g}$ corresponding to a luminous galaxy, a core of filtering scale $R_{c}$ or equivalently, mass $M_{c}$, has undergone nonlinear collapse by some redshift $z_{c}$. The result (Silk and Szalay 1987) is that, provided $M_{c}<<M_{g}$,

$$
\frac{1+z_{c}}{1+z_{g}} \approx\left(\frac{M_{g}}{M_{c}}\right)^{1 / 3}
$$

On scale $R_{g} \approx 1 h^{-1} \mathrm{Mpc}$, the redshift of collapse is $z_{g} \approx 2$. From equation (8), one infers that core collapse of $\sim 10^{9} M_{\odot}$ of cold dark matter would have occurred very early, by $z \sim 20$. Thus it should be possible, in principle, by baryonic dissipation and continued contraction to very high density to form the $\sim 10^{8} M_{\odot}$ black holes that are believed to be the central engines in quasistellar objects and active galaxies long before these systems become visible at $z \lesssim 4$. Infalling gas clouds and stars trapped by encounters with a dense gaseous disk fuel the accretion disk, which is believed to extend for $\sim 10^{13}-10^{14} \mathrm{~cm}$ around the hypothesized supermassive black hole. This fueling is likely to be associated with the continuing collapse and growth of the core.

One cannot distinguish within the limits of the statistics of gaussian fluctuations between the alternative possibilities that the core consists of multiple clumps, or that the substructure is destroyed as the core grows. However this does not really matter for the prediction of protogalactic luminosity. One may simply assume that a substantial fraction of the baryonic component of the non-linear mass at any given epoch is used in star formation. Galaxies in effect form from inside out. The protogalactic luminosity then grows according to equation (8)

$$
\frac{d M_{c}}{d t} \propto t
$$


The initial core, containing $10^{7}-10^{8} M_{\odot}$ of baryons, will efficiently recycle matter and eventually form stars of abundance approaching the nucleosynthetic yield, about one-third solar abundance for a solar neighborhood - type initial mass function (IMF). If the IMF is enhanced in massive stars relative to the local IMF, as seems necessary to account for the observed metallicity towards the centre of our galaxy, the yield will increase, but on the other hand, the increased energy input into the gas will tend to redistribute widely the enriched stellar ejecta. As the core grows, some baryonic infall into the central regions will occur because of gas dissipation after each generation of massive star formation, and the mean abundance of the surviving low mass stars will decrease with galactic radius. It does not seem possible, given the uncertainties in yield and in gaseous dissipation modelling, to provide any precise estimate of the resulting metallicity gradient in the old stellar population. The core builds up an enhanced metallicity, approaching and possibly surpassing the nucleosynthetic yield due to infall of debris from ejecta of massive stars.

This mode of inside-out formation neatly circumvents one of the principal objections to forming stars prior to the epoch $z_{g}$ of collapse of a galactic mass. A dense, metal-rich stellar core forms without requiring that the protogalaxy be predominantly gaseous at this epoch. Since galaxy formation commences very early, at $z \sim 10$, most of the protogalactic luminosity is radiated prior to $z_{g}$. The peak luminosity at $z_{g}$ (which is of order unity) is reduced relative to that in a model in which the protogalaxy was wholly gaseous at $z_{g}$ by a factor of roughly $\exp \left(t_{g} / t_{*}\right)$, where $t_{*}$ is the duration of the vigorous star formation period. Since $t_{*} \lesssim 10^{9} \mathrm{yr}$ in a single burst model of protoellipticals and $t_{g} \sim 10^{10} \mathrm{yr}$, the effective reduction in visibility of multiple burst protogalaxies may be very considerable indeed. It may be necessary to partially suppress long-lived, low mass star formation in these bursts in order for ellipticals to be sufficiently red by the present epoch.

Moreover, the synthesis of heavy elements at large redshift in massive stars strongly suggests that dust grains will be produced early in the course of protogalactic evolution. This means that the emission of a protogalaxy is likely to be strongly modulated by dust. Certainly, Lyman alpha emission will be weak, as indeed is the case for the apparently young metal-poor dwarf galaxies with HII region-like spectra (Hartmann et al. 1984). Moreover, there may well be sufficient dust produced to absorb and reradiate the bulk of the luminosity in the far infrared spectral region near $100 \mu$. IRAS extreme starburst galaxies, whose infrared luminosity may exceed the optical luminosity by a factor of up to $\sim 100$, may conceivably be prototypes of protogalactic starbursts; indeed this will be argued below for other reasons. 


\section{LYMAN ALPHA CLOUDS}

Luminous galaxies are formed from rare $(2.5 \sigma)$ fluctuations according to the normalization of the biased cold dark matter fluctuation spectrum. The fate of many of the recently collapsing run-of-the-mill lower mass fluctuations will be, I shall argue below, to form dwarf galaxies. The least massive fluctuations that collapse early, at $z \gtrsim 4$, have a different fate, however. It is at about this epoch that the intergalactic medium must have become highly ionized. Quasars, now seen out to a redshift of 4.01 , show no absorption trough blueward of the Lyman alpha emission, indicating that the intergalactic medium contains very little atomic hydrogen. Specifically, the most recent study (Steidel and Sargent 1986) of the Gunn-Peterson limit sets an upper limit of 0.05 on the relative continuum depression due to Lyman alpha absorption, and indicates a neutral density $n_{H I} \lesssim 3 \times 10^{-12} \mathrm{~cm}^{-3}$ at $z=3$. Collisional ionization requires a temperature of $\gtrsim 10^{6}{ }^{\circ} \mathrm{K}$, and a correspondingly larger energy input of $\sim 1 \mathrm{keV}$ per baryon is needed to counter radiative energy losses. Photoionization is far less extravagant energetically; since the recombination time is very long, only $\sim 20 \mathrm{eV}$ per baryon is needed at $z \sim 4$. However, the photoionization source is unknown. The recent realization that quasars undergo luminosity (rather than density) evolution limits the ionization efficiency of the ionizing photon flux from quasars, to at most 10 percent of the flux required for IGM ionization if $\Omega_{b} \approx 0.1$ (Shapiro 1986). The most likely source is the ionizing radiation produced by massive stars during the early phases of protogalactic evolution. If the heavy elements seen in extreme population II stars were processed by such stars at $z>4$, then the resulting photon flux is $\sim 100 \mathrm{eV}$ per baryon. The typical photon energy would be $\sim 10 \mathrm{eV}$, and there would clearly be a sufficient number of energetic photons to photoionize the intergalactic medium to the required degree.

This photoionization flux takes time to build up, requiring protogalactic activity, and also could not be effective at heating and maintaining the high ionization fraction while Compton cooling is significant at $z \gtrsim 10$. The luminosity evolution of the quasars most probably monitors and reflects protogalactic activity. Indeed the only possible example of a primeval galaxy was found by searching in the vicinity of, and at the same redshift as, one of the most distant quasars (Djorgovski et al. 1986). Thus a reasonable guess would be that the metagalactic photoionizing flux has developed to its peak value by $z \sim 3$. Later phases of protogalactic activity may well be shrouded by dust.

Consider now the fate of an isolated $1 \sigma$ fluctuation chosen with a filtering scale of $50 \mathrm{kpc}$. This goes non-linear at $z \approx 5$. It will begin collapsing, but becomes photoionized as the metagalactic ionizing flux builds up. The probability of there being a nearby luminous protogalaxy increases rapidly with redshift since these objects are identified with rare fluctuations. Prior to photoionization, the gas is very cold. Trace $\mathrm{H}_{2}$ cooling will maintain the gas temperature at about 1000 
$\mathrm{K}$, and the comoving Jeans length is about $10 \mathrm{kpc}$. Once the gas is photoionized, however, it is heated to about $30,000 \mathrm{~K}$. The Jeans length increases to about 70 kpc, and the gas becomes unbound. These estimates are somewhat modified by allowance for the gravitational effect of the dark matter, the presence of which allows a stable bound state for an isothermal distribution of gas. A stabilized distribution of neutral gas may still become unbound when photoionized.

For filter scales between roughly $10 \mathrm{kpc}$ and $70 \mathrm{kpc}$, the photoionized gas is freely expanding. These expanding clouds are found to have parameters very similar to the Lyman alpha forest clouds seen in absorption towards quasars at $z \gtrsim 2$ (Bond, Szalay and Silk 1987). A grid of one-dimensional hydrodynamic models has been run to obtain cloud density profiles, which are found to be approximately gaussian. For $\nu \approx 1-2$, the cloud HI column densities span the range $10^{13}-10^{16} \mathrm{~cm}^{-2}$ at the onset of photoionization, and the distribution of cloud column densities at a specified redshift is fixed by the range in $\nu$ on given filter scale: it is found to be approximately proportional to $N_{H I}^{-2}$, the high $N_{H I}$ clouds corresponding to higher $\nu$ clouds. The cloud frequency is $\sim 20(30 \mathrm{kpc} / R)^{3}$ $\mathrm{Mpc}^{-3}$, and there is rapid evolution of $N_{H I}$ with redshift, this quantity decreasing as $(1+z)^{6-7}$ due to the free expansion of the clouds and the fact that $N_{H I} \propto \delta^{2}$, where $\delta$ is the cloud overdensity. All of these properties are in good accord with observations of Lyman alpha clouds. These clouds may therefore be the missing link between primordial density fluctuations and galaxies.

\section{DARK HALOS}

The cold dark matter scenario predicts that dark halos should be a universal phenomenon. Detection of a single isolated galaxy with a symmetrical, Keplerian fall-off in its rotation curve would be a serious blow against cold dark matter. In fact, all spiral galaxies studied to date have either flat rotation curves, characteristic of a dark halo, or else asymmetric rotation curves, suggesting of tidal interaction with a neighbor or warping (van Albada and Sancisi 1986). Dwarf irregular galaxies often have rising rotation curves. Asymmetries in the gas distribution can mask the effect of a dark halo.

Observations of elliptical galaxies are consistent with their possessing dark halos. Evidence for dark halos around ellipticals comes from the presence of extensive hot gas bound to ellipticals (Forman et al. 1985), and the modelling of the multiple shells observed at very faint isophote levels (Hernquist and Quinn 1986).

The dark halos that are inevitable in the presence of cold dark matter account in a natural way for the angular momentum of galactic disks, and the amplitudes and the shapes of galaxy rotation curves. In the absence of a dark halo, the specific angular momentum is about an order of magnitude too small to account for that observed in disk galaxies. However dissipative infalling gas torques up 
against the relatively inert cold dark matter as an isolated protogalaxy forms, and after a reasonable collapse factor of about 5-10 in radius results in formation of a rotationally supported disk (Efstathiou and Jones 1980; Fall and Efstathiou 1980).

The specific angular momentum measures the product of characteristic size and maximum rotation velocity. The size of the luminous galaxy is determined by baryonic dissipation, to be discussed in the following section. The maximum rotation velocity $v_{m}$ is determined solely by the depth of the dark matter potential well that constitutes the halo. Biased cold dark matter results in the observed range (100-300 $\mathrm{km} \mathrm{s}^{-1}$ ) for $v_{m}$ in disk galaxies (Frenk et al. 1985). The biasing is crucial for this to work out correctly, for it is the weakened correlations of the dark matter (relative to the luminous matter) on Mpc scales that are critical. The dark matter correlation scale, appropriate to $1 \sigma$ fluctuations is about $3 h^{-1} \mathrm{Mpc}$, equivalent to a velocity of $300 \mathrm{~km} \mathrm{~s}^{-1}$. It is precisely this velocity which is a measure of the potential energy of the dark halo at turn-around, and manifests itself as the velocity dispersion of the dark halo after collapse and virialization have occurred.

The most recent success in the continuing saga of cold dark matter concerns the shape of galactic rotation curves. The rotation curves are flat because secondary infall of cold dark matter accumulates in a quasi-spherical halo with a density profile $\rho \stackrel{\propto}{\sim} r^{-2}$. However the rotation curve predicted for a system containing only cold dark matter would decline gradually to zero at the center of the galaxy. This is quite different from what is observed in disk galaxies where the rotation curve remains flat well within the presumably baryon-dominated halflight radius (typically several $\mathrm{kpc}$ ), and then declines steeply only within a kpc or so of the centre. In our own galaxy, for example, the dark halo only dominates the baryonic disk and bulge components outside the solar circle, yet the rotation curve is flat into a galactocentric distance of about $2 \mathrm{kpc}$.

This apparently paradoxical situation for a non-baryonic halo (Bahcall and Casertano 1985) has now received a simple explanation in the cold dark matter model (Blumenthal et al. 1986). Consider a protogalaxy containing cold dark matter together with a baryonic component amounting to about ten percent of the mass of the system, as expected if $\Omega_{b} \approx 0.1$. The gas dissipates and contracts to form a dense bulge and disk. In doing so, it pulls in some of the dissipationless cold dark matter, which becomes trapped by the locally strong gravity field of the disk. As much as ten percent of the dark matter can be dragged in, and about fifty percent of the spherically averaged density may consist of cold dark matter at one baryonic scale length from the galactic centre. The resulting rotation curve accordingly remains flat well within a baryonic scale length. 


\section{DWARF GALAXIES}

Even dwarf galaxies contain substantial amounts of dark matter. Mass measurements have been performed using HI observations for gas-rich dwarfs and from radial velocities of the brightest stars for nearby dwarf spheroidals. It is found that the ratio of mass to blue luminosity appears to rise systematically with decreasing luminosity, with (Aaronson 1987)

$$
M / L_{B} \propto L_{B}^{-1 / 3} .
$$

This is consistent with the observed surface brightness of dwarfs, for which $L_{B} \stackrel{\propto}{\sim} R^{4}$, provided that the halos all formed at nearly the same epoch, whence $M \stackrel{\sim}{\sim} R^{3}$. One might expect this to arise for low mass galaxies with the cold dark matter fluctuation spectrum.

A simple model which accounts for the decreasing surface brightness and increasing dominance of dark matter towards lower luminosities appeals to baryonic mass loss (Dekel and Silk 1986). Gas outflow is driven by supernovae when the dwarf galaxy is predominantly gaseous, and systematically strips the shallowest potential wells. The stripping occurs because of a galactic wind that arises when supernova remnants overlap before having been decelerated by ambient gas below the escape velocity from the dwarf galaxy potential well. As a consequence of the wind, which arises if the velocity dispersion (one-dimensional) of the dwarf galaxy is below $\sim 60 \mathrm{~km} \mathrm{~s}^{-1}$, the remaining baryons that have already formed stars remain bound but adiabatically expand. The cold dark matter halo helps trap these stars, systematically dominating the shallowest potential wells or least massive systems, which lose proportionally more gas and expand the most. Apart from providing a plausible explanation for the trends in surface brightness and in mass-to-light ratio, this model also accounts for the low metallicities observed for dwarf galaxies. Mass loss means that the process of recycling and progressive enrichment of the gas ejected by massive stars is interrupted, and a systematic decrease of metallicity with decreasing dwarf galaxy velocity dispersion is predicted.

Dwarf protogalaxy stripping also lends to a natural biasing mechanism. A potentially luminous galaxy must satisfy two conditions. One is that its mean dispersion $\sigma$ must exceed $\sim 60 \mathrm{~km} \mathrm{~s}^{-1}$, in order to avoid protogalactic stripping. The second condition is that the mean surface density $\Sigma$ (or equivalently, density) of the old stellar component must exceed a critical value $\Sigma_{c r}(\sigma)$ at any specified value of $\sigma$. This arises because a necessary condition for efficient star formation in a gaseous protogalaxy is that the characteristic energy dissipation time-scale, determined by atomic cooling processes, be shorter than the characteristic dynamical time-scale, typically a crossing time (or a free-fall time) (Silk 1977a; Rees and Ostriker 1977). Whether one considers a uniformly contracting gaseous sphere or, 
more realistically, an ensemble of clouds orbiting and colliding in the protogalactic potential well, one obtains a simple functional form for $\Sigma_{c r}(\sigma)$ that directly reflects the adopted cooling curve (Silk 1983, 1985). The simplest way to derive this result is to note that in order for a shock to be radiative, necessary for strong density enhancement, one has to satisfy the condition

$$
v_{s} t_{\text {cool }} \lesssim R_{\text {cloud }},
$$

where $v_{s}(\propto \sigma)$ is the shock velocity and the postshock cooling time scale $t_{\text {cool }} \approx$ $3 k T_{s}\left(\Lambda\left(T_{s}\right) n\right)^{-1}$ with the postshock temperature equal to $T_{s}$ (proportional to $v_{s}^{2}$ ) and post-shock density equal to $n$. Equation (11) then reduces to a condition of the form $\Sigma_{\text {cloud }} \gtrsim \Sigma_{c r}(\sigma) \equiv v_{s} 3 k T_{s} / \Lambda\left(T_{s}\right)$. Now a necessary condition for effective dissipation triggered by cloud-cloud collisions and ensuing shocks to have occurred during the epoch of formation of the old stars is that the surface density of the old stellar component $\Sigma_{g}$ averaged over the galaxy exceeds $\Sigma_{\text {cloud }}(\sigma)$. This guarantees that a typical cloud collides with another cloud within one mean dynamical timescale for the protogalaxy. Finally, we have $\Sigma_{g}>\Sigma_{c r}(\sigma)$ as a necessary condition for effective protogalactic energy dissipation.

It is straightforward to compare $\Sigma_{c r}(\sigma)$ for a gas of primordial composition with estimates of $\Sigma_{g}$ as a function of $\sigma$ for various galaxy types as well as for galaxy groups and clusters. Data on rotation curves, on the Faber-Jackson relation $\left(L \propto \sigma^{4}\right)$, and on the surface brightness-effective radius relation can be used for the galaxies, and luminosity is converted to mass using values appropriate for the observed stellar population. One immediately notes that galaxies lie in the dissipative regime $\left(\Sigma_{g}>\Sigma_{c r}\right)$, whereas galaxy groups and clusters do not (Faber 1982; Gunn 1982; Silk 1983). This lends some credence to the underlying assumption about the importance of efficient dissipation in protogalactic star formation.

Now $\Sigma_{g}$ represents a fossil memory of the galactic potential well at the termination of the protogalactic phase some $10^{10} \mathrm{yr}$ ago. Apart from rare mergers, little dynamical evolution has subsequently occurred. One would ideally like to compare $\Sigma_{g}$ with the predicted evolution tracks of density fluctuations. This is not presently feasible, but one at least can simply compute the locus of non-linear cold dark matter fluctuations, defined by setting $|\delta \rho / \rho|_{k}=1$ and multiplying the resulting density by a factor appropriate to the collapse and virialization of a spherical shell of cold dark matter. The derived density (or surface density) represents the initial conditions for either $1 \sigma$ or $3 \sigma$ fluctuations that have dissipationlessly collapsed to form a halo of cold dark matter. Within this structure, the baryons can dissipate and contract further to form the dense stellar systems that are the galaxies. Cold dark matter fluctuations are inferred to provide a natural, even if not a unique, framework for the initial conditions that have given rise to galaxies and galaxy clusters (Blumenthal et al. 1984). 
Natural biasing is now seen to arise as a consequence of the joint condition for luminous galaxy formation, namely that proto-galaxies not be stripped and undergo efficient star formation. The $1 \sigma$ fluctuations of galactic mass fail this condition, but a window in the density, velocity dispersion plane opens up for $3 \sigma$ fluctuations that allows luminous galaxies to form. This could explain why the luminous galaxies are more correlated than the underlying matter, the distribution of which is represented by the $1 \sigma$ fluctuations. Dwarf galaxies should therefore be the true representative of the underlying dark matter correlations, and measurement of their correlation length and peculiar velocities would yield an unbiased measure of $\Omega$.

Something is missing from this model, however, for it is well known that different morphological types are systematically correlated with mean density and velocity dispersion. To account for this, on has to examine a further aspect of the galaxy distribution, namely the environment.

\section{ENVIRONMENTAL IMPACT}

The obvious explanation for morphological differences appeals to differences in dissipation rate. To achieve the higher density and binding energy of early type galaxies requires more dissipation. At the same time, there is a seemingly contradictory requirement, namely that the early type systems apparently underwent more efficient star formation than late-type systems. Stars must form early and rapidly to form a spheroid whose structure is largely due to anisotropic but random stellar motions, whereas formation of a thin disk, which is rotationally supported, requires strong gaseous dissipation. One way out of this dilemma is provided by dissipative collapse models (Larson 1975; Carlberg 1984 a,b), which successfully reproduce the luminosity profiles of ellipticals, and allow the possibility via continued infall and dissipation, of accounting for the high core densities. In the cold dark matter scenario of hierarchical formation, similar conditions arise between mergers of gaseous protogalaxies of comparable mass. These mergers largely destroy any preexisting disks, account for the low rotation of ellipticals (Negroponte and White 1983), and preferentially occur in denser regions where the early type galaxies consequently predominate. However mergers are only an effective solution if the galaxies involved are initially gas-rich protogalaxies. Mergers of mature disk galaxies cannot produce the observed frequency of early type galaxies with large bulge-to-disk ratios (Ostriker 1980). Moreover, the star formation rate must be enhanced by the mergers and be efficient, otherwise residual gas will dissipate and contract into a disk that eventually forms stars.

I shall argue that it is the environment that controls the efficiency of star formation. The key to efficiency is the initial mass function. Low mass stars ( $\lesssim 3 M_{\odot}$ ) mostly lock up mass in long-lived remnants whereas massive stars lose mass and recycle enriched matter. High efficiency is achieved if one can have 
many cycles of star formation for a given initial mass of gas, and this requires an IMF enriched in massive stars. The star formation rate can then be high, without prematurely exhausting the gas supply. Precisely this situation is believed to occur in starburst galaxies, where the star formation rate, measured by the far infrared luminosity, is enhanced by up to a factor of 100 relative to the timeaveraged formation rate of the old star component, measured by the blue or visual luminosity (Rieke et al. 1985). The IMF appears to be bimodal even in our own galaxy, where massive star formation seems to be enhanced relative to low mass star formation in the spiral arms (Güsten and Mezger 1983). A bimodal IMF leads to reasonable estimates of the gas depletion time-scale in spiral disks (Sandage 1986), and to a simple explanation of the paucity of metal-poor dwarfs in the solar neighborhood (Larson 1986).

A further clue comes from the observation that starbursts are apparently triggered by tidal interactions between neighboring galaxies (Joseph and Wright 1985). On a milder level of enhancement of star formation rate, spiral arms are of course associated with density waves that enhance the rate of cloud-cloud collisions. It is likely that tidal stirring and enhancement of cloud peculiar velocities leads to cloud growth and that massive molecular clouds are especially favorable sites for massive star formation. One reason may be that such clouds become gravitationally unstable, and crude arguments about fragmentation favor a larger critical mass for the minimum or characteristic fragmentation scale in the warm, relatively massive molecular clouds (Silk 1977b; Larson 1985). Another possibility is that non-linear interactions between HII regions and supernova remnants either induce further massive star formation (Klein et al. 1986) or suppress low mass star formation: such activity is only likely to occur in the more massive clouds where the mass reservoir favors the simultaneous formation of several OB stars.

One can try to relate all of this to protogalaxies by the following speculative reasoning. Star formation rates in protogalaxies can be inferred from population synthesis modelling of the observed spectral energy distribution in elliptical and spiral galaxies (Bruzual 1983). This constrains the low mass star formation rate, that is to say, the stars which contribute to the observed light over time-scales of $10^{8}-10^{10} \mathrm{yr}$. One finds that in a protoelliptical, the star formation rate was about $300 M_{\odot} \mathrm{yr}^{-1}$ over $\sim 10^{9} \mathrm{yr}$ for an $L_{*}$ galaxy $\left(L_{*} \approx 10^{10} h^{-2} L_{\odot}\right)$, declining abruptly thereafter, compared to a rate that is a few percent of this, and relatively constant or even slowly increasing with time, for a spiral or irregulargalaxy where there is continuing star formation over $\sim 10^{10} \mathrm{yr}$ (Gallagher, Hunter and Tutukov 1984). It is tempting to identify protoellipticals with the starburst phenomenon: since with bimodal star formation, the integrated birth rates of massive and low mass star formation are comparable, one finds a comparable enhancement of the specific star formation rate in protoellipticals to that seen in starbursts (Silk 1988). If this analogy holds, then protoelliptical star formation would also be bimodal, 
and correspondingly efficient at recycling gas and rapidly forming the enriched stellar population of the spheroid before dissipation could lead to disk formation. To form a disk apparently requires a continuing gas reservoir over $\sim 10^{10} \mathrm{yr}$, as can occur for isolated galaxies. In galaxy protoclusters, however, the gas supply is likely to be strongly regulated by tidal interactions and collisions between galaxies, as well as by stripping due to hot, intergalactic gas.

However it is not so much the gas supply as the star formation rate that is the primary driver of galactic morphology. Rapid, efficient star formation exhausts the gas supply before a disk can form. Protogalactic mergers may also play a role, but again, the result of a merger will be to temporarily boost the star formation rate. More prolonged stimulation of star formation may arise from tidal heating between protogalaxies in a protocluster, and this could help account for the striking correlation between morphology and local density of galaxies. This effect can be significant only for protogalaxies, which were much larger in cross-section than galaxies are today.

For example, the rate of tidal heating between a galaxy and its neighbors in a cluster can be written

$$
\dot{E} / E=n_{g} \sigma_{E} v_{g}
$$

where $n_{g}$ and $v_{g}$ are the galaxy number density and rms velocity dispersion in the cluster and $\sigma \approx 30 r_{e}^{2}\left(v_{*} / v_{g}\right)^{2}$ is the cross-section for energy exchange in a tidal encounter between identical protogalaxies with de Vaucouleurs profiles, effective radii $r_{e}$, and velocity dispersions $v_{*}$ (Aguilar and White 1985). This expression is appropriate if the protogalaxy consists of an ensemble of many small gas clouds. The tidal heating enhances their mutual collision rate and thereby presumably stimulates star formation, as apparently happens in environments as diverse as spiral arms and in starbursts. Active stirring is guaranteed if the characteristic heating time-scale $E / \dot{E}$ is comparable to the protogalaxy dynamical time-scale. Adopting values characteristic of a protocluster $\left(v_{100} \equiv v_{0} / 100 \mathrm{~km} \mathrm{~s}^{-1}, n_{100} \equiv n_{g} / 100 \mathrm{Mpc}^{-3}\right)$, one finds that the protogalaxy (mass $M_{11} \equiv M / 10^{11} M_{\odot}$ ) binding energy scales with galaxy density as $v_{*}=76 M_{11}^{3 / 5}\left(n_{100} / v_{100}\right)^{1 / 5} \mathrm{~km} \mathrm{~s}^{-1}$. Some further dissipation is required to enhance $v_{*}$ to the value appropriate for a luminous galaxy. This suggests that if tidal heating indeed governs star formation efficiency in a protogalaxy of specified mass, the parameters of the resulting stellar distribution will be such that velocity dispersion increases with increasing density of galaxies, producing higher surface brightness spheroids in denser regions.

Consideration of the observed range of $n_{g}=10^{-2}-10^{4} \mathrm{Mpc}^{-3}$ suggests that relatively cold, and consequently very extended, protogalaxies form slowly in the field, allowing disks to develop and the star formation rate to be low, whereas in dense regions, protogalaxies would be compact, form stars rapidly, and not have 
sufficient time for slow dissipative formation of a stellar disk. Dynamical relaxation should ensure that there is a large dispersion in galaxy parameters about the mean defined by this predicted correlation. By comparison, the observed dependence of morphological type on local density (Dressler 1980) is reasonably consistent with theoretical expectation in the very simple model that I have presented. SO galaxies form an intermediate class, and it may be that mergers in protocluster cores are responsible for converting proto-SO's to protoellipticals. Tidal heating therefore could lead to a natural biasing scheme when galaxies are studied in a volume-limited sample. This mechanism accounts for the dependence of galaxy morphology on luminous galaxy density, because spheroid formation is enhanced in regions where tidal heating stimulated more efficient and vigorous star formation.

\section{CONCLUSIONS}

Cold dark matter provides a remarkably detailed prescription for galaxy formation and for the large-scale structure of the universe. Whether it provides a successful explanation for all of the data is not yet clear. Certainly, there are numerous successes, including on large scales, the uniformity of the microwave background, the correlation function of galaxies, the formation of galaxy groups and clusters, the peculiar velocities of galaxies, and the concentration of luminous matter relative to dark matter. On smaller scales, highlights include explanation of the range of galaxy masses, the density profiles of dark halos and of luminous cores, the shapes of galaxy rotation curves, and some of the characteristics of dwarf galaxies. We do not yet have a good understanding of the origin of the galaxy luminosity function or of the tight correlations found between characteristic parameters of luminous galaxies such as surface brightness, effective radius, and central velocity dispersion. However, refinements in modelling of galaxy formation are likely to soon improve this situation.

Of greater concern is the lack of any explanation in a cold dark matterdominated universe of the cluster-cluster correlations or of the large-scale drift velocities of galaxy clusters. While definitive confirmation of these, still uncertain, observational results would be fatal for the standard model of biased cold dark matter with scale invariant gaussian density fluctuations, there seem to be several possible variations on this theme that may then arise, phoenix-like, in a resurrected theory of large-scale structure. As far as galaxy formation is concerned, however, the current theory appears to be on a more secure footing, in part because many of the observational characteristics of galaxies have been thoroughly studied, and the theoretical goals are well defined. One has the advantage of being able to treat galaxies as fossils, and develop cosmogonical theories to explain the vast array of rich phenomena that we observe in nearby systems. The next major step will be to test these theories by directly searching for young galaxies and for protogalaxies in remote regions of the universe. For the present, however, one may speculate that 
galaxy formation theory will undergo few radical changes despite the uncertainty of our understanding of large scale structure.

This research has been supported in part by grants from NASA and NSF. I am indebted to various colleagues for stimulating and enlightening discussions, including R. Wyse, A. Szalay and M. Davis. 


\section{REFERENCES}

Aaronson, M. 1987, in Nearly Normal Galaxies: From the Planck Time to the Present, ed. S.M. Faber (New York: Springer Verlag) (in press).

Aguilar, L. and White, S.D.M. 1985, Astrophys. J., 295, 374.

Bahcall, J.M. and Casertano, S. 1985, Astrophys. J. Lett, 293, L7.

Bahcall, N. and Soneira, R. 1983, Astrophys. J., 270, 20.

Barnes, J., Dekel, A., Efstathiou, G. and Frenk, C. 1985, Astrophys. J., 295, 368.

Bardeen, J., Bond, J.R., Kaiser, N. and Szalay, A. 1986, Astrophys. J., 304, 15.

Blumenthal, G., Faber, S., Primack, J., and Rees, M., 1984, Nature, 301, 584.

Blumenthal, G., Faber, S.M. Flores, R.A. and Primack, J.R. 1986, Astrophys. J., 301, 27.

Bond, J.R., Szalay, A. and Silk, J. 1987, in preparation.

Bruzual, G. 1983, Astrophys. J., 273, 105.

Carlberg, R. 1984a, Astrophys. J., 286, 403.

Carlberg, R. 1984b, Astrophys. J., 286, 416.

Davis, M., Efstathiou, G., Frenk, C. and White, S.D.M. 1985, Astrophys. J., 292, 371.

Dekel, A. and Silk, J. 1986, Astrophys. J., 303, 39.

Djorgovski, S., Spinrad, H., McCarthy, P., and Strauss, M. 1986, Astrophys. J. Lett. (in press).

Dressler, A., 1980, Astrophys. J., 236, 351.

Dressler, A., Faber, S.M., Burstein, D., Davies, R.L., Lynden-Bell, D., Terlevich, R.J. and Wegner, G. 1987, Astrophys. J. (in press).

Efstathiou, G. and Jones, B.J.T. 1980, Comm. Astrophys., 8, 169.

Faber, S.M. 1982, in Astrophysical Cosmology, ed. H.A. Brück, G.V. Coyne and M.S. Longair (Vatican: Pontificia Academia Scientarum), p. 219.

Fall, S.D.M. and Efstathiou, G. 1980, Mon. Not. Roy. Astr. Soc., 193, 1895.

Forman, W., Jones, C. and Tucker, W. 1985, Astrophys. J., 243, 102.

Frenk, C., White, S.D.M., Efstathiou, G. and Davis, M. 1985, Nature, 317, 595.

Frenk, C., White, S.D.M. and Davis, M. 1983, Astrophys. J., 221, 417.

Fritschi, M. et al. 1985, Physic Letters B, 173, 485.

Gallagher, J.S., Hunter, D.A. and Tutukov, A.V. 1984, Astrophys. J., 284, 544. 
Gunn, J.E. 1982, in Astrophysical Cosmology, ed. H.A. Brück, G.V. Coyne and M.S. Longair (Vatican: Pontificia Academia Scientarum), p. 233.

Güsten, R. and Mezger, P.G. 1983, Vistas in Astronomy, 26, 159.

Hartmann, L.W., Huchra, J.P. and Geller, M.J. 1984, Astrophys. J., 187, 487.

Hernquist L. and Quinn, P. 1986, Astrophys. J. (in press).

Joseph, R.D. and Wright, G.S. 1985, Mon. Not. Roy. Astr. Soc., 214, 87.

Kaiser, N. 1984, Astrophys. J. Letters, 284, L9.

Kaiser, N. and Silk J. 1986, Nature (in press).

Klein, R., Sandford, M.T. and Whitaker, R.W. 1986, Astrophys. J. (in press).

Koo, D. 1986, in Spectral Evolution of Galaxies, ed. C. Chiosi and R. Renzini (D. Reidel: Dordrecht), 419.

Larson, R.B. 1975, Mon. Not. Roy. Astr. Soc., 173, 671.

Larson, R.B. 1985, Mon. Not. Roy. Astr. Soc., 214, 379.

Larson, R.B. 1986, Mon. Not. Roy. Astr. Soc., 218, 409.

Ling, E., Frenk, C. and Barrow, J.D. 1986, preprint (Univ. of Sussex).

Mészáros, P. 1974, Astron. Astrophys., 37, 225.

Negroponte, J. and White, S.D.M. 1983, Mon. Not. Roy. Astr. Soc., 705, 1009.

Ostriker, J. 1980, Comm. Astrophys., 8, 177.

Politzer, H.D. and Wise, M. 1984, Astrophys. J. Letters, 285, L1.

Rees, M.J. and Ostriker, J.P., 1977, Mon. Not. Roy. Astr. Soc., 179, 541.

Rieke, G.H., Catri, R. M., Black, J.H., Kailey, W.F., McAlary, C.W., Lebofsky, M.J. and Elston, R., 1985, Astrophys. J., 290, 116.

Sandage, A., 1986, Astron. Astrophys., 161, 89.

Shapiro, P. 1986, preprint.

Silk, J., 1977a, Astrophys. J., 211, 638.

Silk, J., 1977b, Astrophys. J., 214, 157.

Silk, J., 1977, Astrophys. J., 214, 784.

Silk, J., 1983, Nature, $301,574$.

Silk, J. 1985, Astrophys. J, 297, 1.

Silk, J. 1987, in Star-Forming Regions, ed. J. Jugaku and M. Peimbert (D. Reidel: Dordrecht). 
Silk, J. and Wyse, R.F.G. 1986, in Structure and Evolution of Active Galactic Nuclei, ed. G. Giuricin, F. Mardirossian, M. Mezzetti, and M. Rumella (D. Reidel: Dordrecht).

Silk, J. and Szalay, A. 1987, in preparation.

Steidel, C.C. and Sargent, W.L.W. 1986, Astrophys. J. (in press).

Steinhardt, P. and Turner, M. 1984, Phys. Rev., D29, 2162.

Turner, M. 1985, in Fundamental Interactions and Cosmology, ed. J. Audouze and J. Tran Thanh Van (Editions Frontieres: Gif-sur-Yvette), 267.

Van Albada, T.S. and Sancisi, R. 1986, Phil. Trans. R. Soc. Lond., A000, 000.

White, S.D.M., Frenk, C., Davis, M. and Efstathiou, G. 1987, Astrophys. J. (in press).

Yang, J. et al. 1984, Astrophys. J., 281, 493.

\section{DISCUSSION}

BIRNS: Your model would seem to predict that dwarf galaxies in voids are gas-poor. Is this inconsistent with recent reports by Moody and Kirsher of emission-line galaxies, that would suggest a gas-rich environment in the heart of the Bootes void?

SIIK: I would expect the incidence of gas-rich dwarfs to be much less in voids than elsewhere. Also the emission line galaxies found in the Bootes void are relatively luminous compared to the dwarfs that are unable to retain their gas.

TURNER: You must identify a fluctuation of a particular number of $\sigma$ with an $L^{*}$ galaxy. This must give you three independent quantities: the correct galaxy number density, the appropriate fraction of the Universe's total mass associated with galaxies, and the appropriate mass per galaxy (i.e., internal velocities). How well does this three constraints and one parameter game work?

SIIK: Amazingly, it all works out! The procedure is the following. Fix the correlation length to normalize the fluctuation spectrum and the galaxy number density to specify the number of $\sigma$. Then cosmic coincidence number one is that the cosmic virial theorem yields $\Omega$ (luminous) equal to about 0.1 .

Cosmic coincidence number two is that the biased cold dark matter spectrum of fluctuations simultaneously specifies the correct maximum 
rotational or internal velocities for the dark halos. Specifically, the mass correlation length is unity at a Hubble velocity today of $300 \mathrm{~km} \mathrm{~s}-1$, and this is precisely what is needed to give the binding energy of dark halos that are forming recently.

WEBB: Could you give any further details on the confining mechanism for your Ly $\alpha$ cloud model. Also, are there observational predictions such as a correlation between $\mathrm{N}_{\mathrm{HT}}$ and $\mathrm{b}$ for example? Can any quantitative statements be made concerining the amplitude and scale of any clustering.

SILK: In the Ly $\alpha$ cloud model I described, the absorbing clouds are freely expanding and unconfined. They are more weakly clustered than galaxies。

The correlation length predicted is about one-half of that expected for luminous galaxies at the same redshift. For the low column density clouds (s $10^{14} \mathrm{~cm}^{-2}$ ) the internal velocity dispersion of the cloud is determined mostly by the sound speed $\left(-20 \mathrm{~km} \mathrm{~s}^{-1}\right)$, and should be nearly independent of HI colum density. At higher colum densities, the differential Hubble expansion velocity across the cloud is progressively more important and $v$ should increase by a small factor.

ROTAN-ROBINSON: You are predicting that dwarf el ?ipticals, formed fron 1- $\sigma$ fluctuations, are more uniformly distributed than the 2.5- $\sigma$ giant el1ipticals. What are you predicting about spirals? If they fom from the rare 2.5- $\sigma$ fluctuations, would they not show the same derree of clustering as ellipticals?

SIIK: Spirals and ellipticals alike form from 2.5 o fluctuations. The cluster environment helps convert spirals to elliptical by tidally induced star formation or mergers. Hence the spirals avoid dense regions and are more weakly clustered than ellipticals.

EILIS: Searches for spectacularly luminous primeval galaxies have so far failed and already suggest the formation redshift of such objects would be $z 5$. What is the advantage of invoking a more gradual onset of luminosity in young galaxies?

SILK: If most of the heavy elements seen in the old star components are formed early in the protogalaxy's history when it was still largely gaseous and just an agglomeration of interacting clouds,- then its peak luminosity will be substantially reduced. This means that protogalaxies could be present at low redshift and not be easily detectable for two reasons: first, the predicted fluxes are perhaps an order of magnitude less than expected in standard models, and 
second, the prior phase of heavy element synthesis makes it very likely that they are dusty objects. Perhaps UV or optical wavelengths are not the optimun spectral regions to search for primeval galaxies.

CHFN: (1) That is the life time of the free expansion model of Lya clouds? (2) That is the hehavior of the Lya clouds before the birth of the OSก which provides the energy for the re-ionization?

SIIK: The radius doubling time for a Ly $\alpha$ cloud in our model is about $\sim 2 \times 10^{4} \mathrm{yr}$. Prior to the ionization flash, the potential Ly $\alpha$ clouds are density fluctuations that are just entering the non-linear regime. Smaller scales $\left(\leqslant 10^{7} \mathrm{M}\right)$ may already have collapsed.

GIRAID: What do vou do with those SO galaxies which are not in dense enviroments?

SILK: I presume that protogalaxies are still predominantly gaseous at the onset of galaxy clustering. Hence much of the early star formation can be enhanced or induced by tidal interactions and by mergers. This means that the spheroidal components of galaxies will be largest where star formation is most efficient and most rapid. If this is correct, one can then understand why ellipticals form in the densest regions. The star formation enhancement criterion, however, depends in a systematic way on local density and galaxy peculiar velocity, so that bulge sizes should systematically decrease as one goes from rich clusters to poor clusters and to the field. SO galaxies are therefore an intermediate ponulation that dominate the intermediate range of environment between rich clusters and the field. This distinction is not so rigid however that one would not expect to find some SO's in the extreme environments as well, simply because the local star forming history is presumed to be dominated by stochastic events.

HARTWICK: What are the observational parameters of a primeval galaxy in the cold dark matter scenario?

SILK: The final collapse of the halo of a typical primeval galaxy is very recent (redshift $z \sim 1$ ), but pieces of the primeval galaxy form much earlier. For example, the core collapses as early as $z \sim 10$. This means that the peak luminosity is considerably less (by perhaps a factor of 10) than if the onset of formation was instantaneous. Moreover, the early star formation should produce a considerable amount of dust. A primeval galaxy should be dusty, have a luminosity perhaps 100 times larger than a mature galaxy, and be visible at $z \approx 1-2$. 